Théologiques

Théologiques

\title{
Du corps sauvé au corps lieu de l'expérience de Dieu
}

\section{Jean-Guy Nadeau}

Volume 5, numéro 2, octobre 1997

Le corps : du dualisme à l'altérité

URI : https://id.erudit.org/iderudit/024949ar

DOI : https://doi.org/10.7202/024949ar

Aller au sommaire du numéro

\section{Éditeur(s)}

Faculté de théologie de l'Université de Montréal

\section{ISSN}

1188-7109 (imprimé)

1492-1413 (numérique)

Découvrir la revue

\section{Citer cet article}

Nadeau, J.-G. (1997). Du corps sauvé au corps lieu de l'expérience de Dieu. Théologiques, 5(2), 71-90. https://doi.org/10.7202/024949ar

\section{Résumé de l'article}

On parle souvent d'une dichotomie entre le corps et l'esprit dans la tradition chrétienne. Or, il serait peut-être plus juste de parler d'une subordination du corps à l'esprit, et plus précisément au salut. Le christianisme a en effet toujours considéré le corps comme déterminant pour la relation à Dieu. Ainsi, constitue-t-il un réfèrent théologique de base chez saint Paul et a-t-il toujours été source de préoccupation majeure pour la tradition chrétienne. L'article rappelle des éléments de cette tradition, y situe l'affirmation contemporaine du corps comme lieu de l'expérience de Dieu, et cherche à en identifier les sources et les contours. 


\section{Du corps sauvé au corps lieu de l'expérience de Dieu}

Jean-Guy NADEAU

Faculté de théologie Université de Montréal

\section{RÉSUMÉ}

On parle souvent d'une dichotomie entre le corps et l'esprit dans la tradition chré tienne. Or, il serait peut-être plus juste de parler d'une subordination du corps à l'esprit, et plus précisément au salut. Le christianisme a en effet toujours considéré le corps comme déterminant pour la relation à Dieu. Ainsi, constitue-t-il un référent théologique de base chez saint Paul et a-t-il toujours été source de préoccupation majeure pour la tradition chrétienne. L'article rappelle des éléments de cette tradition, y situe l'affirmation contemporaine du corps comme lieu de l'expérience de Dieu, et cherche à en identifier les sources et les contours.

We often speak of a dichotomy between mind and body in the Christian tradition. Yet, it would be more to the point to speak of a subordination of the body to the mind, and more precisely, to salvation. Christianity has in effect always considered the body as the determinant for the relationship to God. Thus, it constitutes a fundamental theological referent for Saint Paul and it has always been a source of major preoccupation for the Christian tradition. This article recalls the elements of this tradition and situates within it the contemporary affirmation of the body as the place of the experience of God, and looks to identify in it, its sources and contours.

Ce qui vient immédiatement à l'esprit (et au corps) lorsqu'on parle du rapport du christianisme au corps, c'est sa division entre le corps et l'esprit, mais surtout sa méfiance envers le corps, en fait sa mortification du corps - corps de passion, de plaisir et de péché -, et particulièrement sa méfiance envers le corps de la femme, celle "par qui le péché est entré dans le monde . On comprend que plusieurs rejettent cette position et valorisent aujourd'hui, comme le faisait déjà l'Évangile, un rapport plus 
positif au corps et l'identifient comme lieu de l'expérience de Dieu, à tout le moins d'expérience spirituelle.

Il est alors commun de parler du passage d'une vision dichotomique à une vision unitaire du corps et de l'esprit. Or, la tradition chrétienne n'est pas aussi dichotomique qu'on le croit dans son rapport au corps. Il serait en fait plus juste de parler d'une subordination du corps à l'esprit, et plus précisément au salut éternel. S'il paraît nouveau de parler du corps comme lieu de l'expérience de Dieu, et d'abord dans cette catégorie bien contemporaine d'expérience, le christianisme a toujours considéré le corps comme déterminant pour la relation avec Dieu. Rappelons-en quelques exemples: les expériences et les exhortations pauliniennes à glorifier Dieu dans notre corps ( 1 Co 6,$20 ; P h 1,20)$, à y porter l'agonie de Jésus et à y manifester sa vie (2 Co 4,10), à offrir nos corps en hostie vivante, sainte, agréable à Dieu $(R m 12,1)$; la foi à la résurrection de la chair; la conception des sacrements comme signes et voies sensibles de la grâce et du salut; le rôle que les exercices spirituels de saint Dominique et de saint lgnace accordaient au corps non seulement dans l'expression mais dans l'appel, voire la provocation de l'expérience spirituelle; les fresques de la chapelle Sixtine que Jean-Paul II a qualifiée de "sanctuaire de la théologie du corps humain ${ }^{1}$ "; l'Ascension du Ressuscité ainsi que l'Assomption de la Vierge Marie, "montée au ciel avec son âme et avec son corps ».

Mais cet aspect fondamental de la conception chrétienne du corps a été écrasé par une éthique promouvant la maîtrise de l'esprit sur le corps et la mortification de la chair que Foucault considérait comme thème majeur d'une pastorale chrétienne visant le salut, la vie dans l'autre monde ${ }^{2}$. Par exemple, si la dogmatisation de l'Assomption s'opposait à la tendance à mépriser le corps ${ }^{3}$, il s'agit d'un corps idéal dont la virginité signe la participation au salut ${ }^{4}$. Ce qui est loin de l'expérience du corps que réclament nos contemporains. Voyons donc quelques enjeux et aléas du rapport chrétien au corps ou, comme on dit aujourd'hui et peut-être plus justement, des rapports chrétiens aux corps. Nous irons d'abord aux sources de la théologie chrétienne du corps avant d'explorer la théologie du corps comme lieu de l'expérience de Dieu.

1 JEAN-PAul II, Homélie à l'occasion de la réouverture de la chapelle Sixtine, 8 avril 1994, cité dans Le Devoir, 9 avril 1994.

2 Michel FOUCAULT, « Omnes et singulatim: Vers une critique de la raison politique ", Le Débat 41 (1986) 19-20.

3 Bernard HÄRING, Une morale pour la personne. Paris, Mame, 1973, p. 60.

4 Jacques JOUBERT, Le corps sauvé. Paris, Cerf, 1991, p. 9. 


\section{Le corps sauvé}

Caro salutis est cardo (Tertullien ${ }^{5}$ )

C'est à Paul que remonte la théologie chrétienne du corps. Et d'abord dans un texte clé $(1$ Co 6,12-20) qui déterminera la vision chrétienne du corps individuel et collectif, aussi bien que la radicalité des rapports sexuels pour l'identité chrétienne. En fait, le corps constitue un référent théologique majeur chez Paul, pour désigner tant le disciple du Christ que son Église. Elizabeth Schüssler-Fiorenza et Elisabeth Moltmann-Wendel remarquent par exemple avec intérêt que "ce n'est pas l'âme ou la raison ou le plus intime de soi-même, c'est le corps qui est l'image et le modèle de l'être de l'Église ${ }^{6}$ ".

La théologie paulinienne allie de façon originale des éléments de la tradition juive, du stoïcisme et de l'expérience chrétienne de Paul. Je l'aborderai par le biais d'une affirmation selon laquelle celui qui s'unit à la pornè (prostituée, débauchée ${ }^{7}$ ) ne fait avec elle qu'un seul corps, arrachant même les membres du Seigneur pour en faire des membres de pornè (1 Co 6,15-16). Si je pars de cette affirmation c'est parce qu'elle développe un thème qui pose problème à la mentalité contemporaine aussi bien qu'à celle de l'époque: les débauchés (pornoi) sont exclus du Royaume (1 Co 5,$11 ; 6,9$; etc); mais surtout parce qu'elle paraît excessive dans la mesure où elle accorde à l'union sexuelle (même avec une pornè) la même densité qu'à l'union avec le Christ. L'argumentation de Paul est d'autant plus intéressante que nous y trouvons à la fois l'affirmation de l'intimité du corps et de l'âme et celle de la subordination du corps au salut.

\subsection{L'héritage stoïcien contre le corps de passions}

On trouve dans la morale stoïcienne une source de l'argumentation de Paul. 1 Corinthiens s'inscrit dans un monde où l'on considère que l'exercice sexuel, comme l'alimentation, relève de la santé et de la nécessaire satis-

5 «La chair est la charnière du salut. " Voir plus loin, note 38.

6 E. SCHÜSSLER-FIORENZA, En mémoire d'elle, citée dans El. MOLTMANN-W ENDEL, “Esprit et corps. La réponse féministe ", Concilium 265 (1996) 86.

7 Signalons ici la confusion des traductions qui rendent pornè par prostituée, porneia par débauche et pornos par débauché. Ce qui nuit au lien entre ce texte et le problème de 1 Co 5 auquel il répond peut-être; voir R. KLEMPTHORNE, "Incest and the Body of Christ. A Study of 1 Corinthians VI, 12-20», New Testament Studies 14/4 (1968) 568-574. 
faction de besoins naturels ${ }^{8}$. Or la morale stö̈cienne, soucieuse de libérer l'homme de l'asservissement des passions, refuse ces mœurs et tend à inscrire l'exercice sexuel sous le contrôle de la raison qui détermine l'être humain. Celui-ci sera digne à condition que ses passions soient contrôlées par la raison, le plaisir apparaissant comme un dérèglement des sens par lequel l'homme perd sa nature propre ${ }^{9}$.

Ce que nous appelons aujourd'hui le corps de plaisir fait problème puisque y sont liées les passions qui nuisent à l'activité de la pensée. Pis encore, même lorsque la raison arrive à contrôler les passions, "le corps veut sans cesse qu'on s'occupe de lui " (Platon), il interrompt par ses exigences sans fin l'activité du moi pensant et force l'esprit à quitter le Ciel des idées pour redescendre dans la caverne des affaires humaines ${ }^{10}$.

La métaphysique [...] voit l'essence de l'homme dans sa dimension consciente qui trace le domaine du concept, de l'idée, qui l'identifie à l'instance de l'absolu, de Dieu. En face, la phusis, c'est-à-dire le corps, le sensible, sont rejetés dans l'inessentiel: ils sont appelés à disparaître. La vie est dans le suprasensible et la mort du côté de la phusis. [...] Dans ce schéma, Dieu, l'absolu, n'a pas de correspondant dans la phusis ${ }^{11}$.

Christianisant ce thème, Augustin considérera comme "une véritable prostitution que de se séparer du Bien suprême et unique, pour se plonger dans la multitude des joies sensuelles, dans l'amour du siècle et dans la corruption des choses de la terre ${ }^{12} \%$. On connaît cette maxime d'un moine du désert, qui fit fortune : "Quand on veut prendre une ville, on coupe l'eau et les vivres; de même pour les passions de la chair ${ }^{13}$ ».

8 F. HaUCK et S. SCHUlz, dans G. KITTEL, ed., Theological Dictionary of the New Testament, vol. VI. Grand Rapids, Eerdmans, 1968, p. 582; et John L. MCKENZIE, Dictionary of the Bible. Milwaukee, Bruce, 1965, p. 701.

9 Cette position marque toute la tradition chrétienne. Même Thomas d'Aquin, qui reconnaît le plaisir comme intrinsèquement bon puisqu'il vient de Dieu, considère qu'il empêche la personne d'être entièrement au service de Dieu; et cela d'autant qu'il y a une certaine saleté dans la décharge séminale. Voir Mark TOON, The Philosophy of Sex According to St Thomas Aquinas. Washington, Catholic University of America Press, 1954.

10 Hannah A RENDT, La vie de l'esprit. Vol. 2 Le vouloir. Paris, PUF, 1983, p. 50.

11 Yves LEDURE, Si Dieu s'efface. La corporéité comme lieu d'une affirmation de Dieu. Paris, Desclée, 1975, pp. 77-78.

12 Saint AugustiN, "Sermo CXLII », II, 2; Patrologie latine 38, p. 779.

13 Marcel BERNOS et all., Le fruit défendu. Paris, Centurion, 1985, p. 66. 
Dans son souci apologétique d'offrir au monde l'image d'une conduite morale exemplaire, Paul adopte ces vues stoïciennes qu'il soumet toutefois à la dynamique du salut chrétien. Deux maximes stoïciennes pourraient même avoir directement influencé sa position sur la porneia : "L'homme qui a une relation avec des hétaïres pèche contre lui-même » et "Par tout acte impur, un homme souille la divinité en son $\operatorname{sein}^{14}$ \%. Reprises par Paul, elle donnent : "Tout autre péché commis par l'homme est extérieur à son corps. Mais le débauché (pornos) pèche contre son propre corps ", et "Ne savez-vous pas que votre corps est le temple du Saint-Esprit?"

\subsection{Le corps sauvé de la dynamique judéo-chrétienne}

Une seconde source de l'argumentation de Paul tient à l'anthropologie juive qui l'a formé et à la dynamique du salut que Paul a justement expérimenté dans son corps. Confronté à un problème éthique et surtout communautaire, Paul réagirait ici à l'anthropologie gnostique de chrétiens de Corinthe considérant que ce qui touche le corps ne saurait toucher l'âme ${ }^{15}$. Or la tradition juive considère que l'homme est tout un avec son corps. De sorte que le Seigneur qui sauve l'homme le sauve tout entier avec son corps. Objet de l'action divine, ce que nient les gnostiques, le corps s'en trouve radicalement associé à la vie nouvelle et à l'homme nouveau dans le Christ.

Si tout est permis à la liberté chrétienne, tout cependant ne lui convient pas (1 Co 6,12). Non pas en fonction du souci de soi (Foucault), mais en fonction de l'origine de cette liberté dans la mort du Christ et de la relation du disciple avec le Christ Seigneur, relation qui n'échappe cependant pas au souci de soi dans la mesure où il s'agit de son salut. Celui qui a été racheté ("Quelqu'un a payé le prix de votre rachat») ne saurait agir comme s'il était encore esclave c'est-à-dire, en contexte stö̈cien, asservi par ses passions. Ce serait tromper la largesse de son Libérateur, ou de son nouveau maître («vous ne vous appartenez pas »), la réduire à néant. Et comment le chrétien peut-il signifier ce rachat et la gloire de Celui qui est en lui s'il ne le fait dans son corps, dans sa pratique dirions-nous aujourd'hui?

Ultimement, c'est le respect et la réalisation actuelle et eschatologique du corps sauvé que vise Paul. Racheté par la mort du Christ, le corps du chrétien ou de la chrétienne est membre du Christ et temple de

Voir F. HAUCK et S. SCHULZ, op. cit., p. 583.

J. MURPHY O'CONNOR, "Corinthian Slogans in 1 Co 6,12-20 ", Catholic Biblical Quarterly 40 (1978) 394. Voir aussi, Wayne A. MEEKS, The First Urban Christians. New Haven, Yale University Press, 1983, p. 122. 
l'Esprit saint. Augustin en tirera une exhortation significative. Alors que Bulla Regia se prépare à accueillir la foire avec son cortège de courtisanes, il prêche à ses ouailles :

O frères de Bulla [...] Vous êtes les membres du Christ [...] Irais-je prendre les membres du Christ pour en faire les membres d'une prostituée? Et nos chrétiens, non seulement donnent leur cœur à des prostituées, mais ils entraînent dans cette souillure des femmes qui en étaient indemnes - comme si ces créatures n'avaient point d'âme; comme si le sang du Christ n'avait pas encore été répandu pour elles aussi, comme si l'Ecriture ne déclarait pas: les courtisanes et les publicains vous précèdent dans le Royaume des Cieux ${ }^{16}$.

Mais pourquoi la relation sexuelle apparaît-elle aussi radicalement déterminante pour le corps et sa relation au Christ? Paul trouve son argumentation du côté de Genèse 2,24 : "Ne savez-vous pas que celui qui s'unit (du verbe kollâ̂ ) à la prostituée fait avec elle un seul corps (soma)? Car il est dit: "Les deux ne seront plus qu'une seule chair" (sarxa) ". Le verbe kollaô (attacher, souder) indique l'attachement définitif de l'homme à la femme, voire l'attachement au Seigneur ${ }^{17}$. Quoiqu'en une occasion le terme qualifie la fréquentation des prostituées $(S i$ 19,2), il renvoie habituellement à une union radicale que marque bien l'idiome juif traduit par chair (que Paul modifie ici en soma) qui "signifie en réalité 'vraie vie humaine'18 ${ }^{\prime 1}$. Une seule chair, un seul corps c'est donc une seule vie. Bien que Paul précise que "celui qui s'unit avec le Seigneur fait avec lui un seul esprit ${ }^{19}$ ", on ne saurait donc concevoir l'union au Christ comme relevant du spirituel et l'union sexuelle comme relevant du physique. Le corps signe et signifie toute la personne. D'où la résurrection du corps.

Un dernier principe, suggéré plus haut, éclaire la position de Paul : «Ne savez-vous pas que votre corps est le temple (naos) du Saint Esprit qui est en vous? » Le corps est ainsi considéré comme le naos, le Saint des saints, c'est-à-dire "la partie la plus reculée du sanctuaire qu'habite le

16 Saint Augustin, Sermones, XVII, 7, Denis, 1792.

17 Voir Dt 10,20;11,22; 2 R 18,6; Jr 13,11; etc.

18 F. R. BARRY, A Philosophy from prison, 1926, p. 151; repris par R. BATEY, «The mia sarx union of Christ and the Church ", New Testament Studies 73/3 (1967) 279.

19 Marquant l'étonnement devant cette chute du texte alors qu'on attendrait « un seul corps", la note de la TOB en conclut que Paul "veut éviter que ce réalisme soit compris de façon trop grossière ". 
Dieu ${ }^{20}$ ». D'où l'importance, préciserons-nous dans le contexte de cette réflexion, de l'intégrité du corps, indisssolublement physique et spirituelle, l'une témoignant de l'autre. De là la conception patristique que "nul vase d'or ou d'argent n'est aussi cher à Dieu que le temple d'un corps virginal $^{21}$ \#. De sorte que, "pour les femmes comme pour les hommes, le salut dépend surtout de la chasteté que nous aurons fait paraître [il est donc lié au corps]. Nous sommes le Temple de Dieu où le Saint Esprit a consacré sa demeure, et la chasteté est gardienne et prêtresse de ce temple ${ }^{22}$ ».

La chasteté corporelle des chrétiens, et surtout des chrétiennes, constitue une valeur tellement radicale « que pour nous, affirme encore Tertulien, la perte de la chasteté est plus affreuse que la peine de mort la plus cruelle 23 ». C'est ainsi que Jean Chrysostome louera la jeune Pélagie qui, "sur le conseil et avec l'assistance de Jésus-Christ ", a préféré se donner la mort en se précipitant du haut du toit paternel - certains y verront un suicide ou une mort assistée - plutôt que d'être dénudée et peutêtre violée par les soldats venus l'arrêter :

Douleurs, tortures, affreux supplices, elle était prête à tout supporter, mais elle craignait de perdre la couronne de la virginité. [...] Donc, elle refusa d'entrer au tribunal, de s'exposer en spectacle à la licence des regards; de permettre aux désirs impurs de jouir de son aspect; elle mit son corps sacré à l'abri des outrages; de la chambre virginale, du gynécée, elle passa dans un autre asile de la chasteté, dans le $\mathrm{ciel}^{24}$.

En faisant un modèle pour ses ouailles, comme on le fera plus tard de Maria Goretti, Chrysostome conclut ainsi une autre homélie en l'honneur de la sainte ${ }^{25}$, où on retrouve nettement le motif stoïcien : «Imitons donc, nous aussi, sa modestie, sa continence, et dressons des trophées de nos

Osty, "Première épître aux Corinthiens ", cité par René POTHIER, Une étude exégétique de 1 Co 6,12-20. Mémoire de licence en théologie, Université de Montréal, 1973, p. 97.

21 Saint JÉrome, "Lettre à Eustochium », Patrologie latine 22, p. 394-425.

22 TERTULLIEN, cité dans France QUÉRÉ-JAULNES, La femme. Les grands textes des Pères de l'Église. Paris, Centurion, 1968, p. 142. Voir aussi le texte de Jean CHRYSOSTOME cité en p. 182 du même ouvrage.

23 Tertullien, Apologétique L 12, Paris, Belles Lettres, 1929, p. 108.

24 Jean Chrysostome, « Homélies sur sainte Pélagie. Première homélie ". CEuvres complètes III. Toulon, Guérin, 1864, pp. 498-499.

25 Bien que sa canonisation par la piété populaire sera critiquée par certains pasteurs, dont Augustin, elle paraît néanmoins révélatrice de l'époque. 
victoires sur les voluptés; réprimons la fougue de nos désirs déréglés, effrénés $26 \%$.

On ne saurait ici passer sous silence le statut du corps féminin dans la théologie du corps élaborée par des hommes chastes ou visant la chasteté. $\mathrm{Du} \mathrm{III}^{\mathrm{e}}$ au $\mathrm{XX}^{\mathrm{e}}$ siècle, la théologie a largement été «l'œuvre de moines pour qui la femme symbolise ce à quoi ils ont renoncé et qui menace sans cesse leur attachement à Dieu. [...] Pour eux, l'altérité de la femme signalerait plutôt l'altérité du diable 27 ! ». Associée au plaisir et aux passions, voire au sexe ("les personnes du sexe»), la femme menace l'attachement à Dieu. Fille d'Eve, réceptrice et réceptacle du stupre (pourtant masculin) par lequel Augustin, suivi par la tradition, considérait que se communique la faute originelle, la femme s'en est trouvée marquée comme l'autre dont on doit se protéger. Et s'il faut la protéger elle aussi, comme l'a fait la pastorale des Pères, ce sera le plus souvent sous le mode de l'enfermement.

\subsection{Le corps menacé par autrui}

Les travaux de Gilbert Durand, de Mary Douglas et de Vladimir Jankelevitch permettent de saisir les motifs anthropologique de base de ces discours. Durand signale par exemple que le schéma corporel constitue le premier modèle anthropologique pour la symbolisation non seulement de l'espace mais aussi de la relation au transcendant ${ }^{28}$. Pour Douglas, la forme extérieure est la condition même de l'existence et de l'identité, aussi bien en religion qu'en société ${ }^{29}$. D'où le fait que l'exigence de sainteté doit s'exprimer de façon physique. Ce n'est qu'à ce niveau, en effet, que la sainteté peut être attestée aussi bien par le sujet lui-même que par les contrôleurs sociaux et donc ecclésiaux. Commentant 1 Corinthiens 5 et 6 , Wayne Meeks signale que sainteté et impureté y sont comme des catégories opposées, celle d'impureté apparaissant comme une métaphore désignant des liaisons sexuelles interdites ${ }^{30}$. C'est ainsi

26 Jean Chrysostome, «Homélies sur sainte Pélagie. Deuxième homélie ", op. cit., p. 500 .

27 Eric FUCHS, Le désir et la tendresse. Genève, Labor et Fides, 1979, p. 110. Voir aussi Michel Legrain, Le corps humain. Du soupçon à l'évangélisation. Paris, Centurion, 1978, p. 189.

28 Gilbert DURAND, Les structures anthropologiques de l'imaginaire. Paris, P.U.F., 1960, pp. 443-444. Pour une perspective plus culturelle et critique, voir Paula M. COOEY, Religious Imagination and the Body. A Feminist Approach. Oxford et New York, Oxford University Press, 1994.

29 Mary Douglas, Purity and Danger. London, Rootledge \& Kegan, 1966, p. 81.

W.A. MEEKS, The First Urban Christians, p. 130. 
qu'aujourd'hui encore, les divorcés remariés ne peuvent communier que si "ils assument l'engagement de vivre une totale continence 31 ». Certes, on ne parle plus d'impureté, mais cette norme laisse voir combien l'instance sexuelle, et donc le corps, reste déterminante pour l'identité chrétienne, comme si certains y situaient le noyau du symbole de l'union du Christ et de l'Église.

L'interaction étroite entre la métaphore ecclésiale du corps (dont on voit les pièges) et l'anthropologie chrétienne ${ }^{32}$ réside peut-être en partie dans le fait que la pensée chrétienne sur le corps s'est élaborée à une époque où le corps chrétien est persécuté, contesté dans son intégrité physique aussi bien que sociale. Or les conditions sociales d'un petit groupe minoritaire persécuté "se prêtent bien aux croyances qui symbolisent le corps comme un contenant imparfait qui ne sera rendu parfait que s'il devient imperméable 33 ". Encore une fois, les études de Meeks corroborent celles de Douglas : "les admonitions de 1 Corinthiens 5 et 6 présupposent une conception de la communauté comme un espace pur et saint, et du monde extérieur comme impur et profane ${ }^{34}$ ».

Jankelevitch considère que c'est parce que le corps est l'instrument des promiscuités et des relations avec l'autre, et ainsi suspect d'impureté, que la pureté est souvent identifiée à la pureté corporelle. À cause de sa qualité homéostatique, tout contact, même superficiel, est considéré dans l'ordre de la pureté comme une pénétration profonde ${ }^{35}$.

De ce qui précède, on comprend que pureté et virginité, son corrollaire attestable, soient devenues les qualifications par excellence du corps, temple de l'Esprit saint. Encore récemment, un théologien pourra affirmer sans problème que «seul Dieu est l'Autre que nous pouvons pénétrer sans atteindre des limites décevantes et qui peut pénétrer en nous sans nous

31 JEAN-PAUL II, «Discours de clôture du synode romain sur le mariage et la famille (25 octobre 1980)", cité dans Informations catholiques internationales 556 (1980) 40.

32 Voir Jean-Guy NADEAU, La prostitution, une affaire de sens. Montréal, Fides, 1987, pp. 251-276; et « Inculturation of Ecclesiology and Morals through Pastoral Ministry toward Prostitutes ", Catholic Theological Society of America Proceedings [Louisville (KY)] 45 (1990) 92ss.

33 M. DOUGLAS, Purity and Danger, p. 158.

34 W.A. MEEKS, The First Urban Christians, pp. 100-101.

35 Vladimir JANKelevitch, Le pur et l'impur. Paris, Flammarion (Champs), 1960, pp. 61-62. 
violer, nous aliéner ${ }^{36}$ ". Réflexion semblable, mais concernant cette fois le toucher oculaire, de la part de Jean-Paul II qui, louant le corps humain, affirmait néanmoins que "si on le sépare de cette dimension [de foi], il devient d'une certaine manière un objet, facilement avili, puisque ce n'est qu'aux yeux de Dieu que le corps humain peut rester nu et découvert et conserver sa splendeur et sa beauté ${ }^{37}$ ».

\subsection{La chair et l'ame unies dans le service et la récompense}

C'est ainsi que si le christianisme a intimement uni le corps et l'esprit (ou l'âme) c'est souvent davantage comme des vases communicants que comme l'entité indivise qu'on en fait aujourd'hui. On en trouve un bel exemple chez Tertullien ( $\mathrm{II}^{\mathrm{e}}$-III ${ }^{\mathrm{e}}$ siècles) qui considérait le corps, la chair comme "la charnière du salut ".

La chair est la charnière du salut. De sorte que, lorsque l'âme est choisie par Dieu pour le salut, c'est la chair qui fait que l'âme peut ainsi être le choix de Dieu. Ainsi, la chair est lavée pour que l'âme soit purifiée; la chair reçoit l'onction pour que l'âme soit consacrée; la chair est marquée d'un signe pour que l'âme soit protégée; la chair est couverte de l'ombre de l'imposition des mains pour que l'âme soit illuminée par l'esprit; la chair se nourrit du corps et du sang du Christ pour que l'âme se repaisse de la force de Dieu. On ne peut donc pas les séparer dans la récompense puisque le service les réunit. Car même les sacrifices agréables à Dieu [...] c'est la chair qui les offre à son propre préjudice ${ }^{38}$.

Bien que leur union dans la récompense marque leur indissocialibilité, certains noteront la différence radicale entre ces affirmations et l'Évangile selon lequel ce n'est pas ce qui pénètre en l'homme qui le rend impur (ou pur?), mais ce qui sort de son cœur (Mt 15,17-20; Mc 7,15). La direction de ce vecteur physique de purification (et de contagion) constitue l'un des problèmes majeurs du rapport de la tradition chrétienne au corps.

On ne saurait donc affirmer que cette tradition dissocie le corps et l'esprit. Bien au contraire, on y trouve l'affirmation de leur union radicale : ce qui touche, souille ou purifie l'un touche, souille ou purifie l'autre. Entre le monde et l'âme, tout ou presque tout passe par le corps.

Jean-Guy PAGÉ, Réflexions sur l'Église du Québec. Montréal, Bellarmin, 1976, pp. 76-77.

37 Jean-Paul 11, "Discours de clôture du synode romain... " .

38 TERTULLIEN, La résurrection de la chair, VIII; Patrologie latine 2, colonne 806. 
D'où la nécessité d'exercer le plus grand contrôle sur le corps qui y circule, d'autant que la vie éternelle y est en jeu. C'est ainsi que la tradition chrétienne subordonne toujours le corps (et donc le sujet) à quelque réalité qui le transcende, tels le salut éternel, l'autorité qui en définit la gestion, et plus récemment l'amour (mais sans passion ${ }^{39}$ ). Entre le corps et Dieu se dresse alors l'écran de l'autorité (absolue) et de l'institution, dont on sait combien elles font problème aujourd'hui. C'est en partie sur ce fond de scène que se pose l'affirmation, qui en apparaît revendicatrice, du corps comme lieu de l'expérience de Dieu.

\section{Le corps, lieu de l'expérience de Dieu}

L'enjeu de la vie spirituelle réside dans le corps. C'est là que se fait l'expérience de Dieu, là que Dieu se révèle à l'âme, là qu'il parle au cœur humain, là qu'il prend son repas avec lui $(A p 3,20)^{40}$.

Ce qui précède permet de saisir l'intérêt que la tradition chrétienne accorde au corps sauvé ou, plus humblement, " charnière du salut ». Va-ton plus loin lorsqu'on en parle comme lieu de l'expérience de Dieu, en fait de l'expérience que nous faisons de Dieu?

Désigner ainsi le corps c'est évoquer diverses réalités. C'est prendre acte de l'union intime du corps et de l'esprit que notre culture a remise à l'avant plan, et saisir l'expérience humaine en fonction de cette conception. C'est référer aux diverses expériences de la transcendance, aux expériences sommet (peak experiences) qui ont à l'évidence un ancrage corporel, et considérer l'expérience comme lieu théologique, voire théologal. C'est considérer l'Évangile comme récit non seulement de l'incarnation et de la résurrection, mais de la pratique historique, donc corporelle, du «Fils de Dieu». C'est sans doute aussi emboîter le pas à une culture qui refuse la mortification du corps au nom du salut dans l'au-delà.

Sur la toile de fond de ce qui précède, c'est sûrement aussi rejeter la main-mise de l'institution ecclésiale sur le corps et le schéma métaphysique ou onto-théologique qui la justifiait. Le corps s'en trouve marqué d'un signe positif, reconnu capable de soi et de l'autre, de communication, de soins, d'amour, de reproduction (ou de procréation, dont le terme dit bien la grandeur de la chose en la reliant à l'expérience de Dieu). Quoiqu'il en

39 Puisque désirer sa femme avec passion c'est la réduire au rang d'objet (voir Jean-Paul Il, À l'image de Dieu homme et femme. Paris, Cerf, 1980, p. 142ss; et Le corps, le cour et l'esprit. Paris, Cerf, 1984, p. $101 \mathrm{ss).}$

40 Richard Bergeron, « Le corps, lieu de la rencontre de Dieu. Perspective chrétienne ", Nouveau Dialogue. Le Corps comme Lieu de Spiritualité, Hors série jeunesse, automne 1996, p. 29-30. 
soit de ses possibilités de manipulation, on considére par exemple le plaisir sexuel non plus comme dérèglement de la raison, mais comme ouverture et sortie de soi, confiance et abandon, en somme don à l'autre. De même que nos prédécesseurs avaient construit le corps comme lieu du péché et la condition corporelle comme lieu de ce qui éloigne de Dieu, on le construit aujourd'hui comme lieu de l'expérience de Dieu et de la grâce.

\subsection{Le corps du sujet}

La sensibilité moderne face au corps s'inscrit dans la foulée des affirmations de Nietzsche, rejetant le mépris du corps dans la philosophie : "Corps suis je, tout et totalement, et rien outre; et âme n'est qu'un mot pour désigner quelque chose dans le corps ${ }^{41}$ \%. Pour certains, corps et esprit ne sont pas des réalités différentes, mais des signes, "des mots utilisés pour distinguer divers attributs de la même substance. Une personne est cette sorte d'être dont on peut parler à la fois en termes de corps et d'esprit 42 ». L'affirmation du corps comme lieu de l'expérience de Dieu apparaît alors comme le choix d'un autre signe linguistique que ceux de l'âme ou du cœur pour dire le lieu de cette expérience, voire cette expérience elle-même. Ce choix apparaît de plus comme un geste politique affirmant non seulement l'unité du corps et de l'esprit, mais réclamant une nouvelle autorité du corps et une vie meilleure en ce monde. "Adieu la Raison, bonjour la Vie ${ }^{43}$."

"Je ne sais pas si j'ai une âme, mais un corps, pas de doute!" Plusieurs tiennent de tels propos où l'on reconnaît la valorisation du temporel que Guy Rocher caractérisait comme le principal changement culturel de notre époque ${ }^{44}$. Du motto "Nous n'avons qu'une âme et nous devons la sauver ", notre culture est passée à "Nous n'avons qu'un corps et nous devons le sauver». Jadis objet de mépris, le corps « est devenu objet de salut. Il s'est littéralement substitué à l'âme dans cette fonction

41 F. NIETZSCHE, Ainsi parlait Zarathoustra. Des contempteurs du corps, cité dans Yves LEDURE, Si Dieu s'efface, p. 39.

42 Michel BERNARD, "De la corporéité comme "anticorps" ou de la subversion esthétique de la catégorie traditionnelle de «corps» ", dans Catherine GARNIER, dir., Le corps rassemblé. Pour une perspective interdisciplinaire et culturelle de la corporéité. Montréal, UQAM, éd. Agence d'Arc, 1991, p. 33.

43 Richard Gendron, "Adieu la Raison, bonjour la Vie. Discussion sur la connaissance, la culture et le paradigme écologique ", communication au Congrès de l'ACFAS 1997.

44 Guy ROCHER, "La révolution tranquille des valeurs ", Le Souffle 4/21 (mars 1968). 
morale et idéologique 45 ». Désormais l'ascèse ne se tient plus contre le corps, mais avec et souvent pour lui. Et Baudrillard poursuit, évoquant une page publicitaire de la revue Elle: "Si vous ne faites pas vos dévotions corporelles, si vous péchez par omission, vous serez punies. Tout ce dont vous souffrez, c'est par irresponsabilité coupable envers vous-même (votre propre salut) $[\ldots]$ ici ce n'est plus Dieu qui vous punit, c'est votre propre corps 46 ".

Il s'en suit cependant que ce corps qui venait tout juste d'échapper à la raison se trouve à nouveau rationalisé, fonctionnalisé en valeur d'usage, valeur d'échange économique et marchande et en valeur/signe : "Je suis mon corps" se transforme souvent en "Je vaux mon corps... regardez-le, voyez-moi; aimez-le, aimez-moi. "Ainsi sommes nous passés de l'exigence d'une tête bien faite à celle d'un corps bien fait, bien vêtu. Douce revanche du corps : l'apparence et non plus la raison, comme on le voit en politique, est devenue pour plusieurs le critère d'évaluation du discours. Or l'apparence est coûteuse, de sorte que si la libération du corps sert la séduction, elle sert aussi largement la consommation 47 .

Le salut s'est donc sécularisé, corporéisé. Dans une réflexion sur l'étatisation du pouvoir pastoral, Michel Foucault signalait qu'on est passé

du souci de conduire les gens au salut dans l'autre monde à l'idée qu'il faut l'assurer ici-bas. Et dans ce contexte, le mot 'salut' prend plusieurs sens [dont on notera le caractère corporel] : il veut dire santé, bien être (c'est-à-dire niveau de vie correct, ressources suffisantes), sécurité, protection contre les accidents 48 .

"Corps suis je» est donc entré dans les mœurs et a trouvé en Occident son heure de gloire dans les années 1970. En témoigne le succès du titre Ourbodies, Our Selves. Le contexte de cet ouvrage issu d'un groupe féministe indique bien la portée politique de son affirmation qui s'est transformée dans le slogan: "Mon corps m'appartient ", trahissant ainsi l'intuition de Nietzsche citée plus haut. En effet, «s'il m'appartient,

45 Jean BAUDRILLARD, La société de consommation. Paris, Gallimard, 1970, p. 200.

46 J. BAUDRILLARD, La société de consommation, p. 202.

47 J. BAUDRILlARD, La société de consommation, p. 210-211.

48 Michel FoucAulT, « Pourquoi étudier le pouvoir : la question du sujet ", dans $\mathrm{H}$. DREYFus et P. RABINOW, Michel Foucault. Un parcours philosophique (avec un entretien et deux essais de Michel Foucault). Paris, Gallimard, 1984, p. 306. 
c'est avant tout que je suis plus qu'un corps. C'est que je suis aussi une raison, un cœur, une liberté, une Histoire ${ }^{49}$ ".

Le corps apparaît comme ce qui est le plus intime, le plus personnel à l'individu; sûrement plus que ses idées. De sorte que la reconstruction du corps se situe sur le régistre de la subjectivité. Mais le corps étant d'évidence socialisé, cette reconstruction du corps se situe aussi sur les régistres de l'autorité et du droit (concepts peu corporels, on en conviendra, mais issus des pratiques). Chacun, chacune se réapproprie, contre l'institution sociale ou religieuse, l'autorité sur son corps, le droit (et le pouvoir) d'en disposer comme il ou elle l'entend sur les diverses scènes de l'existence : droit au plaisir, droit à l'intégrité corporelle (droit de dire oui et de dire non à une relation sexuelle ${ }^{50}$, mais aussi à un examen médicolégal), droit à la dignité, à la santé, etc. D'où les problèmes liés par exemple à l'identité du fotus : excroissance du corps maternel (et en cela la mère a droit sur lui) ou corps propre en devenir (et alors il faut en protéger les droits).

Mais alors que l'on a rejeté les contraintes de la tradition chrétienne sur le corps, on prend de plus en plus conscience que les requêtes actuelles portent elles aussi leur part de contraintes, voire de moralisme : haro sur celui ou celle qui se laisse aller, c'est-à-dire qui ne prend pas soin de son corps, qui ne le contrôle pas (bien sûr, on n'utilise pas ce terme) par une bonne alimentation, le work out ou le jogging, les crèmes solaires ou anti-rides, l'abstention de tabac ou d'alcool, etc.

Dans notre culture sécularisée, c'est donc le rapport à soi, peut-être la divinité introuvable dont parle Ferry ${ }^{51}$, qui fonde la propriété et la dignité du corps, et non plus le rapport à Dieu. Si l'affirmation du corps comme lieu de l'expérience de Dieu relève en partie de la sécularisation, celle-ci y est revue et corrigée: parler du corps comme lieu de l'expérience de Dieu, c'est ouvrir le corps sur autre chose, sur plus grand que lui, sur plus grand que le sujet humain.

49 Gisèle HaLImI, "La liberté des libertés", dans Choisir de donner la vie. Paris, Gallimard (Petite Bibliothèque Payot 420), 1979.

50 Julie BRICKMAN, « Feminist, Nonsexist, and Traditional Models of Therapy: Implications for Working with Incest ", Women and Therapy 3/1 (Spring 1984) 49-67. Dans une finale non sans résonnance évangélique, l'auteure affirme qu'en brisant le lien entre sexualité et domination, une nouvelle sexualité peut émerger pour tous, où les relations ne seront plus basées sur le pouvoir, la coercition ou l'obligation, mais sur le choix, le droit de dire non, la possibilité d'élargir la joie et le privilège de dire oui.

51 Luc FERRY, L'homme-Dieu ou le Sens de la vie. Paris, Grasset, 1996, p. 172. 


\subsection{Le corps, appel et expérience de la transcendance}

Avec la théorie de la relativité, la science a marqué la détermination majeure que le corps de lobservateur, d'abord par sa position physique, exerce sur la connaissance ${ }^{52}$. Le travail de l'esprit dépend donc intimement de la position du corps. Ce que signalaient déjà, bien que dans un autre domaine, saint Dominique et saint Ignace, et plus récemment un Dechanet (La Voie du silence), dans leurs exercices spirituels. En même temps, de nombreux travaux ont révélé le corps comme premier lieu des apprentissages du sujet, de son image de soi et de sa relation au monde ${ }^{53}$. Ainsi avons-nous pris une vive conscience de l'inscription sexuelle de notre expérience et de nos représentations.

L'expérience spirituelle n'a donc rien à voir avec une quelconque désincarnation. Elle est enracinée, au plus profond, dans le corps, les émotions, la parole, la loi, l'histoire. On ne construit pas sa spiritualité seul mais toujours en dialogue avec sa culture et son temps, son environnement humain aussi bien que physique. Il n'y a pas de vie spirituelle pour les humains que nous sommes (sans préjuger de ceux que nous serons dans l'au-delà) sans les médiations du corps, de la parole, de l'histoire. Dieu est pour moi si j'en fais l'expérience, et tel que j'en fais ou que j'en ai fait l'expérience, une expérience dont la dimension corporelle n'est plus à démontrer.

Nous avons déjà signalé la portée du corps poétique, du corps comme matrice des représentations (Durand). En témoignent en négative les prisonniers en isolement sensoriel qui en perdent tout repère pouvant équilibrer la pensée, voire l'identité 54 . En témoigne en positive une Thérèse d'Avila qui a utilisé un langage très corporel pour dire des expériences pourtant extatiques dont, au début tout au moins, la dimension sprituelle et la dimension corporelle étaient indivisibles. Pensons simplement à la célèbre transverbération mise en scène par Bernini.

Le psychanalyste Jacques Durandeaux signalait que la restauration du corps dans le personnalisme chrétien s'était faite au prix de la méconnais-

52 D'où la crise de la représentation et de la référentialité, signale M. FouCAULT dans Les mots et les choses.

53 Pensons à Alexander Lowen, Ashley MOntague, Moishe Feldenkrais, Stanley KALEMAN, Jacques DropsY et autres.

54 François JАСOB, Cahiers de la commission médicale de la FIACAT, 1985, cité dans Fêtes et saisons 513, mars 1997, p. 9. 
sance de la pulsion de mort ${ }^{55}$. Mais le corps n'est pas marqué que de cette pulsion et certains jugent à revers que «la corporéité est traversée, animée par un désir $[\ldots]$ de permanence personnelle ${ }^{56}$ ", d'où émergerait une invocation de la transcendance 57 . Ainsi Yves Ledure considère-t-il le corps comme " le point de départ du désir religieux [...] parce que la mort en figure la menace radicale ${ }^{58}$ ». La transcendance et la religion seraient donc appelées par le corps contre sa propre mort, l'au-delà apparaissant alors comme l'eschatologie du corps. On peut par ailleurs se demander si le souci contemporain du corps ne joue pas en partie dans notre culture sécularisée le rôle que jouait autrefois la religion quant à la mort.

Le corps est aussi saisi comme lieu d'expérience de Dieu à partir de l'extase, des expériences sommets (amour, danse, grossesse, accouchement, orgasme, plongée, ascension, joie, communion, douleur) dont il est le lieu. Sa puissance esthétique le pousse souvent en effet du côté de la transcendance. Espace d'élévation et d'ouverture de l'humain, en même temps que sa limite, le corps vaut bien une cathédrale ${ }^{59}$, image d'autant porteusc quie " le Très Haut n'habite pas des demeures construites par la main des hommes " (Ac 7,48) mais la communication des humains dont le corps est la voie 60 .

$\mathrm{Si}$ elles sont expérience de la transcendance, les expériences sommets ne sont pas de soi expériences de Dieu, bien qu'elles puissent y mener ou la signer. Elles n'en sont souvent qu'un pas, celui de la manifestation qui, en christianisme comme en judaïsme, appelle proclamation 61 . Aussi faut-il, dans la quête de transcendance et l'extase du corps, se garder du rêve d'une relation immédiate avec Dieu qui tenterait par

Jacques DuRANDEAux, Chrétiens au feu de la psychanalyse. Paris, Gallimard, 1972, p. 169.

56 Yves LeDURE, Transcendances. Essai sur Dieu et le corps. Paris, DDB, 1989, p. 115.

Y. LEDURE, Transcendances, p. 173.

Y. LEDURE, Transcendances, p. 157.

59 Je me souviens d'une méditation sur le corps animée par Matthew Fox où nous avions pu contempler la magnificence du corps (au moins égale à celle du cosmos) à travers ses réseaux sanguins, neurologiques, cellulaires, etc.

60 La Cour Suprême du Massachusetts déclarait le 14 mai 1997 que la loi antimendicité de l'État " brimait la liberté d'expression des plus pauvres [de même que] le droit d'aborder les gens dans l'espoir de recevoir aide et compassion ". (L'Itinéraire 4/8, Montréal , août 1997, p. 4).

61 Voir Paul RiCcEUR, "Manifestation et proclamation ", dans E. CASTELLl, dir., Le sacré. Paris, Aubier-Montaigne, 1974, pp. 57-76. 
exemple de dénier la séparation d'avec la mère ou identifierait le salut à l'envers de notre condition actuelle.

L'expérience a besoin de la parole, elle-même enracinée dans le corps et la communauté, pour se construire comme "expérience de Dieu ". Le corps à corps de Jacob avec l'ange ne prend sens que dans la parole; l'épreuve de Job y trouve sinon sens du moins résolution; de même les actes des prophètes, les guérisons évangéliques et de façon plus évidente encore les apparitions du Ressuscité sont-elles accompagnées de la Parole qui les nomme. Autant d'expériences corporelles qui ne sont expériences de Dieu que par la parole et la foi.

\subsection{Le corps évangélique}

Les chrétiens fondent l'affirmation du corps comme lieu d'expérience de Dieu sur l'incarnation et la résurrection du Christ, et par extension "la résurrection de la chair " dont celle du Christ serait la prolepse. Des hommes et des femmes ont fait de Jésus Christ une expérience corporelle... qui les a transformés : "Ce que nous avons entendu, ce que nous avons vu de nos yeux, ce que nous avons contemplé et que nos mains ont touché du Verbe de vie... nous vous l'annonçons. " (1 Jean 1,1-3) Mais peut-on y fonder l'affirmation du corps comme lieu d'expérience de Dieu? Certes l'incarnation a rendu possible une expérience corporelle, historique de Jésus Christ à ses contemporains. Mais est-ce bien notre situation? Pouvons-nous revivre ce qu'ont vécu les contemporains de Jésus?

D'autre part, "l'Incarnation affirme théologiquement ou si l'on préfère théoriquement la pertinence de l'attente religieuse au cœur de la cor-

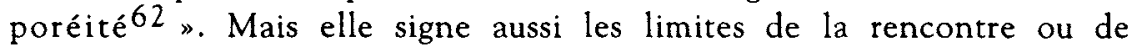
l'expérience de Dieu. C'est dans l'épaisseur du corps plutôt que dans l'immatérialité, la transparence ou la maîtrise de l'esprit que nous pouvons faire l'expérience de Dieu. Ce dont Madame Edwarda de Bataille se veut une illustration saisissante ${ }^{63}$. L'expérience que nous faisons de Dieu à travers "ce corps de vie, ce corps blessé qui est le nôtre ${ }^{64}$ ", fut-il cathédrale, ne saurait qu'être à taille humaine, tissée à même les limites, le clair-obscur, les incertitudes de notre existence, le plus souvent loin de toute évidence voire de tout absolu.

63 Georges BATAILlE, Madame Edwarda. Paris, J.J. Pauvert, 1956; voir par exemple la p. 34 dans l'édition 10/18, 1973.

64 Voir Guy LAPOINTE, "La légèreté de l'Évangile ", Présence Magazine (mai 1997), p. S-4. 
Si la théologie classique accordait un large poids aux souffrances du Christ dans l'économie du salut, on parle davantage aujourd'hui de la pratique de Jésus. Avec l'incarnation, apparaissent le corps sauveur et le corps sauvé. D'une part, l'action salutaire de Jésus vient de son corps: corps de parole aussi bien que d'agir et de toucher, corps crucifié aussi bien que corps de puissance. D'autre part, elle touche le corps. Jésus enseigne, sauve et manifeste le salut à travers des gestes significatifs qui concernent le corps.

Mais ici non plus, l'agir, le toucher ne sont pas tout. Ils sont accompagnés de la parole. Jésus enseigne et son enseignement gestuel et discursif est parfois reconnu. La guérison d'une femme courbée un jour de sabbat l'illustre bien: "Il lui imposa les mains; aussitôt elle redevint droite et se mit à rendre gloire à Dieu. " (Luc 13,13) Guérie dans son corps lié depuis 18 ans, cette femme prend la parole dans l'assemblée et signe l'expérience de Dieu qu'elle vient de faire. Mais comment l'auraitelle pu si elle n'avait déjà connu (et d'abord culturellement) ce Dieu qui sauve, qui guérit? Si le corps est lieu d'expérience de Dieu, c'est donc d'un corps vivant et cultivé qu'il s'agit, d'un corps inséré dans une communauté, communauté aussi nécessaire au corps qu'à la parole comme leur lieu d'émergence, d'expérience, de circulation, de salut.

Aujourd'hui encore des chrétiens recourrent à cette puissance salutaire du Christ et font la file dans divers sanctuaires pour toucher un crucifix ou une statue ${ }^{65}$. Et si la personne historique de Jésus Christ n'est plus présente parmi nous, la foi chrétienne affirme que nous en faisons la rencontre à travers les autres. À travers le service et le soin, c'est l'agir corporel qui s'affirme lieu de la rencontre de Dieu, et non seulement l'affect auquel pourrait se limiter l'expérience. Le chrétien ne saurait séparer l'amour de Dieu de l'amour du prochain. C'est ce à quoi appellent le Sermon sur la montagne et Matthieu 25 qui tirent le corps au-delà de ses expériences de transcendance spontanées et le tournent vers les pratiques de donner, faire du bien, avoir compassion.

Dans l'expérience de Dieu, le système du don se substitue alors au système de la pureté qu'entretenait l'image du corps temple. Bien que le système du don ait toujours été présent à la tradition, présidant par exemple à l'institution du diaconat aussi bien qu'à l'annonce de la Parole, il a peu marqué le discours sur le corps à travers la tradition. Si une large part des pratiques chrétiennes prenait soin des corps, il est clair que ce n'est pas ce vecteur qu'a privilégié le discours, enfermant trop de gens 
dans une expérience qui, loin de les rapprocher de Dieu, les en éloignait. Le récit suivant, qui rapporte l'expérience spirituelle d'une victime de viol, illustre la valeur actuelle de la catégorie d'expérience pour parler de la relation à Dieu, alors que celle de pureté en fermait la voie.

During the rape she became convinced that she would die and resigned herself to her impending death. But when the rapist left and she found herself still alive, prone on the ground, she experienced all around herself a sudden and compelling vision of Christ as a crucified woman. As she lay transfixed by this vision an enormous relief swept over her and she realized that she would not have to explain to a male God that she had been raped. God knew what it was like to be a woman who had been raped 66 .

On ne saurait par ailleurs passer sous silence, malgré leur utilisation répressive ${ }^{67}$, l'importance que la tradition accorde à la participation du corps souffrant aux souffrances rédemptrices du Christ.

Enfin, le corps de Jésus Christ porté par l'Évangile c'est aussi l'Eucharistie. On trouve dans une théologie de l'Eucharistie dont le substantialisme fait aujourd'hui problème un souci d'une corporéisation (en fait de matérialisation) de la personne de Dieu lui-même, à tout le moins du Fils. Si la transubstantiation est si importante dans cette théologie, c'est non seulement parce que l'hostie consacrée présentifie le Christ (une conception symbolique de la chose y suffirait), mais parce que le substantifiant, elle lui donne un caractère de réalité plus fort et permet au fidèle d'avoir une relation corporelle directe avec Lui, d'être vraiment (c'est-à-dire corporellement) proche de Lui.

En Jésus Christ, résume un théologien, «le corps qui sauva est tout à la fois le corps historique troué sur la croix, le corps sacramentel brisé dans l'Eucharistie, le corps mystique divisé dans ses membres 68 ". Les qualificatifs qu'il donne à ce corps, par ailleurs aussi ressuscité et monté au ciel, sont intéressants dans la mesure où ils valent aussi pour notre propre corps, lui aussi troué, brisé, divisé. Ce que prend en compte le dis-

Rosemary REDFORD-RUETHER, "Feminism and Jewish-Christian Dialogue", dans John HICK et Paul F. KNITTER, ed., The Myth of Christian Uniqueness : Toward a Pluralistic Theology of Religions. Maryknoll, Orbis, 1987, p. 146-147. Merci à Louise Melançon d'avoir porté ce texte à notre connaissance.

67 Voir par exemple Joanne C. BROWN et Carole R. BOHN, ed., Christianity, Patriarchy and Abuse. A Feminist Critique. New York, Pilgrim Press, 1989; et Jean-Guy N ADEAU, "Éducation chrétienne et réaction à l'inceste ", Prêtre et Pasteur, 94/5 (1991) 276-286. 
cours sur le corps comme lieu de l'expérience de Dieu. Nous le disions plus haut, cette expérience ne se tient pas dans l'évidence que l'on attribuait autrefois à une révélation qui peinait et qui peine encore trop souvent à emprunter d'autres voies corporelles que celles de la soumission (homonymie du oui et de l'ouie qui en est le premier vecteur corporel), mais dans le clair-obscur des événements, des interprétations et des décisions qui tissent l'expérience humaine.

\section{La passion de l'expérience}

On pourrait sans doute dire de l'expérience de Dieu ce que Heidegger affirme de l'expérience :

Expérimenter quelque chose, cela veut dire: s'acheminant, arriver à atteindre quelque chose sur un chemin. [... ] que cela vers quoi en cheminant nous tendons afin de l'atteindre, cela nous attire nousmême, nous touche, s'adresse à nous et nous met en demeure - en nous retournant jusqu'à nous rendre comme lui. [...] Faire une expérience avec quoi que ce soit, une chose, un être humain, un dieu, cela veut dire: le laisser venir sur nous, qu'il nous atteigne, nous tombe dessus, nous renverse et nous rende autre. Dans cette expression, faire ne signifie justement pas que nous sommes les opérateurs de l'expérience; faire veut dire ici, comme dans la locution 'faire une maladie', passer à travers, souffrir de bout en bout, endurer, accueillir ce qui nous atteint en nous soumettant à lui ${ }^{69}$.

Le discours sur le corps comme lieu de l'expérience de Dieu apparaît donc comme fruit des développements de la connaissance ainsi que comme requête d'autorité de la part du sujet. Bien qu'en d'autres termes et avec des caractères souvent différents, ce discours relève aussi de la tradition chrétienne qui, sans dissocier le corps et l'esprit marque nettement l'autorité de celui-ci sur celui-là et ne manque pas d'ambiguïtés quant au corps sauvé (et donc en rapport avec Dieu). Par ailleurs, en parler en termes d'expérience pourrait, en même temps que rapprocher le salut de chacun et chacune, renouveler la conscience du caractère souvent troublant et immaîtrisé (le tremens et fascinans du sacré) de l'expérience de Dieu que le christianisme occidental récent semble avoir édulcorée.

69 Martin HeIDEGGER, Acheminement vers la parole. Paris, Gallimard, 1976, pp. 161s et 143 . 